\title{
Solution to vitamin B3 mystery challenge
}

\section{Lucia D'Ulivo ${ }^{1}$}

Published online: 1 January 2019

(C) Springer-Verlag GmbH Germany, part of Springer Nature 2018

The winner of the Vitamin B3 mystery challenge (published in volume 410 issue 17) is

Martijn van Fassen, University Medical Center Groningen, University of Groningen, Groningen, The Netherlands.

The award entitles the winner to select a Springer book of his choice up to a value of $100,-$.

Our Congratulations!

\section{Solution}

Pellagra, a disease provoked by chronic deficiency of niacin, became an epidemic in both the USA and Europe as a consequence of a diet based mostly on corn [1]. Strangely, corn contains significant levels of niacin. Moreover, among populations of Mesoamerica, where corn has been a staple food for centuries, pellagra has never been an issue.

In Mesoamerica, and particularly in Mexico, corn is subjected to alkaline treatment (limewater wash) prior to milling. This process is called nixtamalization and has several benefits to the final product. Nixtamalization promotes the elimination of the hull and the dissolution of hemicellulose, which is the main building material of cell walls [2]. The corn can be easily milled and the final flour acquires different visco-elastic properties, which makes it suitable for kneading. Last, but not least, the alkali treatment releases niacin that is bound to the starches [3].

After the discovery of America by Europeans, corn was imported as a new crop to Europe but the alkali treatment was disregarded by Europeans. Although free niacin is

This article is the solution to the Analytical Challenge to be found at https://doi.org/10.1007/s00216-018-1093-8

Lucia D'Ulivo

lucia.dulivo@gmail.com

1450 Sultan Street, ON K4A 0K2, Ottawa, Canada

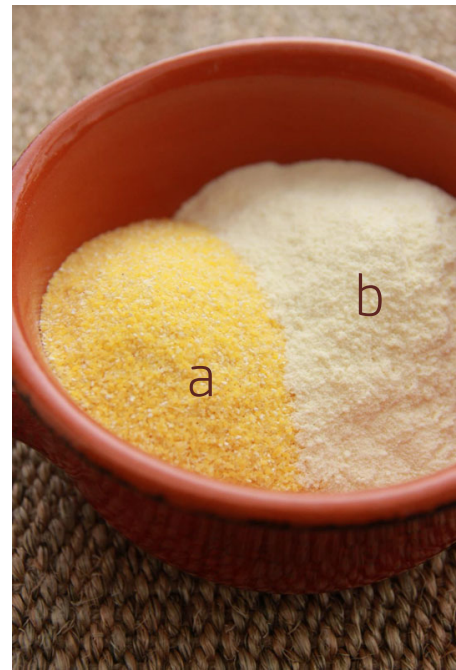

Fig. 1 Regular $\mathbf{a}$ and alkali-treated $\mathbf{b}$ corn flours

found in corn, most of it is bound to starches [3]. The starchbound niacin is not bioavailable, meaning that humans cannot absorb it. Even the acidic environment of the stomach does not release niacin from starches. The only effective method is alkali treatment (Fig. 1).

If the process of nixtamalization would have been imported to Europe along with corn, the spread of pellagra could have been avoided. This also explains why Mesoamericans have never developed the disease.

\section{References}

1. Rajakumar K. Pellagra in the United States: a historical perspective. South Med J. 2000;93:272-277.

2. Sefa-Dedeh S, Cornelius B, Sakyi-Dawson E, Afoakwa EO. Effect of nixtamalization on the chemical and functional properties of maize. Food Chem. 2004;86(3):317-324.

3. Carpenter. The relationship of pellagra to corn and the low availability of niacin in cereals. Experientia Suppl. 1983;44:197222. 